\title{
Role of PTCH1 gene methylation in gastric carcinogenesis
}

\author{
YUN ZUO, YU SONG, MIN ZHANG, ZHEN XU and XIAOLAN QIAN \\ Department of Oncology, The First Hospital of Zhangjiagang, Zhangjiagang, Jiangsu 215600, P.R. China
}

Received September 25, 2013; Accepted April 24, 2014

DOI: $10.3892 / 01.2014 .2178$

\begin{abstract}
The present study aimed to investigate the role of PTCH1 methylation in gastric carcinogenesis and the therapeutic effect of the methylation inhibitor, 5-aza-2'-deoxycytidine (5-aza-dC), in the treatment of gastric cancer. Total RNA was extracted from 20 gastric cancer tissues, their corresponding adjacent normal tissues and a gastric cancer AGS cell line. PTCH1 mRNA expression was detected by quantitative PCR, and the PTCH1 methylation of the promoter was examined by methylation-specific PCR. The AGS cells were treated with 5-Aza-dC; apoptosis and the cell cycle were examined by flow cytometry, and the PTCH1 methylation level was observed. PTCH1 expression was negatively correlated with promoter methylation in the gastric cancer tissues, their corresponding adjacent normal tissues and the gastric cancer AGS cell line $(\mathrm{r}=-0.591, \mathrm{P}=0.006)$. 5-Aza-dC treatment caused apoptosis and the $G_{0} / G_{1}$ phase arrest of the AGS cells, and also induced the demethylation and increased expression of PTCH1. In conclusion, the study found that the hypermethylation of the PTCH1 gene promoter region is one of the main causes of low PTCH1 expression in AGS cells. Demethylation agent 5-Aza-dC can reverse the methylation status of $\mathrm{PTCH} 1$ and regulate the expression of PTCH1, indicating its potential role in gastric cancer treatment.
\end{abstract}

\section{Introduction}

The hedgehog (Hh) signaling pathway is one of the most significant molecular mechanisms for the regulation of the embryonic developmental process, and its activation shows an association with the emergence of a number of solid tumors $(1,2)$. Studying the function and regulatory mechanism of the $\mathrm{HH}$ signaling pathway may be further elucidate the mechanisms underlying the development of malignant tumors and aid in their diagnosis and treatment. The Hh family mainly includes SHH, HHIP, PTCH1, Smo and Gli, where PTCH1 is a negative regulatory factor of the Hh signaling pathway. The Hh pathway could be

Correspondence to: Dr Yun Zuo, Department of Oncology, The First Hospital of Zhangjiagang, 68 West Jiyan Road, Zhangjiagang, Jiangsu 215600, P.R. China

E-mail: zuoyun1@163.com

Key words: gastric cancers, $\mathrm{PTCH} 1$ gene, hedgehog signal pathway, methylation, 5-aza-2'-deoxycytidine activated by inhibiting the expression of PTCH1 and could also be involved in tumorigenesis. It has been reported that the PTCH1 gene could be methylated and involved in the formation of certain tumors (3). However, the association between PTCH1 methylation and gastric cancer is rarely reported. The purpose of the present study was to investigate the role of PTCH1 hypermethylation on gastric carcinogenesis by observing PTCH1 gene methylation and expression in gastric cancer tissues and the gastric cancer AGS cell line, as well as by investigating the effect of the demethylating agent, 5-aza-2'-deoxycytidine (5-Aza-dC), on PTCH1 gene methylation and expression in gastric cancer cells.

\section{Materials and methods}

Specimens and cell culture. A total of 20 gastric cancer tissues and their corresponding adjacent normal tissues were collected from 20 gastric patients who underwent curative resections. These cancer tissue specimens and adjacent normal tissue specimens were routinely confirmed by biopsy and stored in liquid nitrogen. The study group consisted of 14 male and 6 female patients, with a median age of 60.12 years. No primary tumors from other sites were observed for these gastric patients. Prior to surgery, the gastric patients received no other treatment. The human gastric cancer AGS cell line was purchased from the cell center of Shanghai Life Science Institutes of the Chinese Academy of Sciences (Shanghai, China) and was cultured with $10 \%$ fetal bovine serum (Hyclone, Shanghai, China) and Ham's F12K medium Sigma-Aldrich (St. Louis, $\mathrm{MO}, \mathrm{USA})$ at $37^{\circ} \mathrm{C}$ and $5 \% \mathrm{CO}_{2}$. This study was performed at the First Hospital of Zhangjiagang (Zhangjiagang, China) and also approved by the Institutional Review Board of the First Hospital of Zhangjiagang. Written informed consent was obtained from each patient.

Instruments and reagents. 5-Aza-2'-deoxycytidine (5-Aza-dc) was purchased from Sigma-Aldrich, and TRIzol reagent was bought from Invitrogen Life Technologies (Carlsbad, CA, USA). The RNA reverse transcription kit, propidium iodide (PI) and Annexin-V/PI double-staining streaming apoptosis detection kits were purchased from Shanghai Jingmei Biological Engineering Co., Ltd. (Shanghai, China). The methylation conversion kit, EZ DNA Methylation-Gold ${ }^{\mathrm{TM}}$, was purchased from the Beijing Science and Technology Development Co., Ltd. (Beijing, China). The ABI7500 Real-Time PCR instrument was manufactured by Applied Biosystems (Life Technologies, Carlsbad, CA, USA). 
Table I. Primer sequences and length of PCR product.

\begin{tabular}{lllc}
\hline PCR type & Primer name & \multicolumn{1}{c}{ Primer sequences } & Product length, bp \\
\hline qPCR & PTCH1 & 5'-TGTGCGCTGTCTTCCTTCTG-3' & 119 \\
& & 5'-ACGGCACTGAGCTTGATTC-3' & 260 \\
& $\beta$-actin & 5'-GCCATCCTGCGTCG-3' & 197 \\
MSP & 5'-TGGGCACCGGAACCGCT-3' & 197 \\
& Methylation & 5'-GTTAATTCGTGATTTTCGGA-3' & \\
& & 5'-ATAACAAACCTACGAACCGC-3' & \\
& Unmethylation & 5'-AATGTTAATTTGTGATTTTTGGA-3' & \\
\hline
\end{tabular}

qPCR, quantitative PCR; MSP, methylation-specific PCR.

AGS cell treatment with $5-A z a-d C$. AGS cells $\left(3 \times 10^{5}\right)$ were plated in 100-ml flasks with Ham's F12K medium containing $10 \%$ fetal bovine serum at $37^{\circ} \mathrm{C}$ with $5 \% \mathrm{CO}_{2}$. Next, $24 \mathrm{~h}$ after the cells had reached the logarithmic growth phase, they were treated with $5 \times 10^{-6} \mathrm{~mol} / 15$-Aza-dc. The treatment medium was changed every $24 \mathrm{~h}$ for 3 times, then the cells were collected. Control cell groups without 5-Aza-dc treatment were also collected for comparison.

Total RNA extraction, cDNA synthesis and DNA extraction. Total RNA (dissolved with $50 \mu \mathrm{l}$ Tris-EDTA) was extracted by a conventional method once $100 \mathrm{mg}$ tissue in liquid nitrogen had been ground into a powder, or after $1 \times 10^{6}$ AGS cells had been washed with phosphate-buffered saline (PBS) and collected by centrifugation at $12,000 \mathrm{rpm}$ for $15 \mathrm{~min}$. The concentration and purity of the RNA were tested by electrophoresis. To synthesize cDNA, $1 \mu 10.5 \mu \mathrm{g} / \mu \mathrm{l}$ Oligo-(dT) $)_{18}$ and $10 \mu \mathrm{l}$ DEPC $-\mathrm{H}_{2} \mathrm{O}$ were added to $1 \mu \mathrm{l}$ total RNA and mixed, and then bathed in $70^{\circ} \mathrm{C}$ water for $5 \mathrm{~min}$ and cooled rapidly on ice. The following reagents were then added: $4 \mu 15 \mathrm{X}$ reaction buffer, $1 \mu 120 \mathrm{U} / \mu 1$ RNA enzyme inhibitors and $2 \mu 1$ $10 \mathrm{mmol} / \mathrm{ldNTP}$. The mixture was held in a water bath at $37^{\circ} \mathrm{C}$ for $5 \mathrm{~min}$, then $1 \mu \mathrm{l} 200 \mathrm{U} / \mu \mathrm{l}$ reverse transcriptase (M-MuLV) was added and the mixture was placed in a $42^{\circ} \mathrm{C}$ water bath for $60 \mathrm{~min}$. The process was completed with immersion in a $70^{\circ} \mathrm{C}$ water bath for $10 \mathrm{~min}$. The synthesis reaction was terminated. Synthesized cDNA product were stored at $-20^{\circ} \mathrm{C}$. The DNA of the tumor tissues and cells were obtained using the phenol/chloroform extraction method. DNA quality was measured with a NanoDrop-1000 full wavelength UV/VIS scanning spectrophotometer (Thermo Fisher Scientific, Waltham, MA, USA).

PTCH1 gene quantitative (q)PCR analysis. PTCH1 gene qPCR analysis was started with $2 \mu \mathrm{cDNA}$ product as a template, adding $0.5 \mu \mathrm{l}$ for each PTCH1 upstream and downstream primer (Shanghai Sangon Biological Engineering Technology and Services Co, Ltd., Shanghai, China; Table I), as well as $10 \mu \mathrm{l}$ 2X SYBR Green Real Time PCR Master Mix liquid (Shanghai GeneCore BioTechnologies Co., Ltd., Shanghai, China) and $7 \mu \mathrm{l}$ sterile water. Next, detection of 35 amplification loops were conducted with the ABI7500 Real-Time PCR instrument at conditions of $95^{\circ} \mathrm{C}$ for $5 \mathrm{sec}, 55^{\circ} \mathrm{C}$ for $5 \mathrm{sec}$ and $72^{\circ} \mathrm{C}$ for $30 \mathrm{sec}$. The melting curves of the amplified products were analyzed, using $\beta$-actin as an internal reference. The relative expression of the PTCH1 gene was calculated by $2^{-\Delta \Delta \mathrm{Ct}}$ and analyzed by agarose gel electrophoresis.

Cell cycle and apoptosis detection by flow cytometry. Treated and untreated AGS cells $\left(\sim 1 \times 10^{6}\right.$ cells each) were collected and centrifuged at $1000 \mathrm{rpm}$ for $5 \mathrm{~min}$, and then the culture medium was discarded. The cells were washed once with $3 \mathrm{ml}$ $0.01 \mathrm{~mol} / \mathrm{l} \mathrm{PBS}$ (pH 7.4), which was then removed by centrifugation at $1,500 \mathrm{rpm}$ for $10 \mathrm{~min}$. The cells were fixed for $24 \mathrm{~h}$ at $4^{\circ} \mathrm{C}$ by adding $1 \mathrm{ml}$ ice-cold $70 \%$ ethanol. The fixative was centrifuged at 1,500 rpm for $10 \mathrm{~min}$ and discarded again. The cells were resuspended with $3 \mathrm{ml}$ PBS for $5 \mathrm{~min}$. The cells were filtered once with a 400-mesh screen and the PBS was removed by centrifugation. The cells were stained with $1 \mathrm{ml}$ PI dye (final concentration at $1,500 \mathrm{rpm}$ for $10 \mathrm{~min}$ of $100 \mu \mathrm{g} / \mathrm{ml}$, $0.01 \mathrm{~mol} / \mathrm{l} \mathrm{PBS}, \mathrm{pH}$ 7.4) and stored at $4^{\circ} \mathrm{C}$ in the dark for $30 \mathrm{~min}$. A flow cytometer was used to detect the cell cycle and apoptosis (BD FACSCalibur, BD Biosciences, Franklin Lakes, NJ, USA).

DNA bisulfite conversion. DNA bisulfite conversion was conducted using the EZ DNA Methylation-Gold kit, according to the manufacturer's instructions. Briefly, $130 \mu \mathrm{l} \mathrm{CT}$ Conversion Reagent was added to $20 \mu \mathrm{l}$ DNA (500 ng), then mixed and maintained at $98^{\circ} \mathrm{C}$ for $10 \mathrm{~min}$. The sample was then held at $64^{\circ} \mathrm{C}$ for $2.5 \mathrm{~h}$. Storage was at $4^{\circ} \mathrm{C}$. A total of $600 \mu \mathrm{l}$ M-Binding Buffer was added into an activated Zymo-spin column. The converted DNA that was stored at $4^{\circ} \mathrm{C}$ was then added into the spin column and the content was mixed by inversion. Centrifugation at full-speed $(12,000 \mathrm{rpm})$ was applied for $30 \mathrm{sec}$, then the effluent was removed and $100 \mu \mathrm{l}$ M-Wash Buffer was added into the column. Centrifugation at 12,000 rpm was applied for $30 \mathrm{sec}$ and then $200 \mu \mathrm{l}$ M-Desulphonation Buffer was added into the column prior to storage for 15-20 min and centrifugation again at full speed $(12,000 \mathrm{rpm})$ for $30 \mathrm{sec}$. A total of $200 \mu \mathrm{l}$ M-Wash Buffer was added into the column and centrifugation at 12,000 rpm was applied for $30 \mathrm{sec}$. Another $30 \mu \mathrm{l}$ M-Elution Buffer was added, and then the transformed DNA was collected by centrifugation at 12,000 rpm and stored at $-20^{\circ} \mathrm{C}$ for 1 week.

Methylation-specific PCR (MSP) detection. CpG island analysis and primer design for PTCH1 mRNA of the 


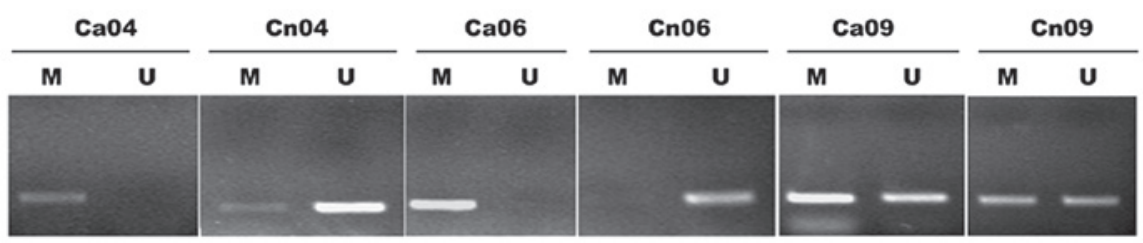

Figure 1. Products of methylation-specific PCR (MSP) analysis of PTCH1 gene promoter methylation in gastric cancer and adjacent normal tissues. Ca, gastric cancer; Cn, adjacent normal tissue; M, methylated PCR products; U, unmthylated PCR products.
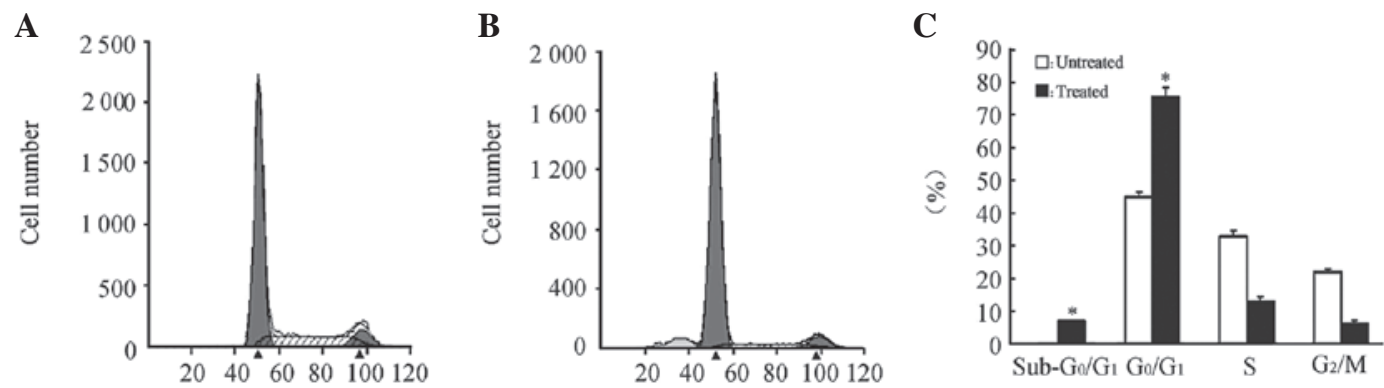

Figure 2. Cell cycle changes in gastric cancer AGS cells treated with 5-Aza-dC, as detected by flow cytometry. (A) Untreated cells. (B) Treated cells. (C) The percentage of cells within the different phases of the cell cycle (" $\mathrm{P}<0.05$ vs. untreated cells; $\mathrm{n}=3$, mean \pm standard deviation). 5-Aza-dC, 5-aza-2'-deoxycytidine.

transcription start site (counted as 0:00) -3950 bp upstream and $+2050 \mathrm{bp}$ downstream were conducted with Methyl Primer Express ${ }^{\circledR}$ v1.0 software (Applied Biosystems; Life Technologies). Table I lists the MSP primers. PCR cooling amplification was performed in $8 \mu \mathrm{l}$ bisulfite-treated DNA with a conventionally configured reaction system. Briefly, pre-degeneration was completed at $95^{\circ} \mathrm{C}$ for $5 \mathrm{~min}$. Prior to PCR, predegeneration was completed at $95^{\circ} \mathrm{C}$ for $5 \mathrm{~min}$. Next, cooling PCR was performed for 10 cycles (PCR degeneration at $94^{\circ} \mathrm{C}$ for $30 \mathrm{sec}$, with renaturation temperature decreases of $0.5^{\circ} \mathrm{C}$ per cycle; temperature decreases from $+3^{\circ} \mathrm{C}$ to $-2^{\circ} \mathrm{C}$ for $30 \mathrm{sec}$; and extension at $72^{\circ} \mathrm{C}$ for $30 \mathrm{sec}$ ). Next, the normal PCR was performed for 40 cycles (PCR degeneration at $94^{\circ} \mathrm{C}$ for $30 \mathrm{sec} ;-2^{\circ} \mathrm{C}$ renaturation for $30 \mathrm{sec}$; and extension at $72^{\circ} \mathrm{C}$ for $30 \mathrm{sec}$ ). Amplification products were analyzed by $1.5 \%$ agarose gel electrophoresis.

Statistical analysis. Data were analyzed with SPSS 13.0 statistical software (SPSS, Inc., Chicago, IL, USA). A non-parametric Mann-Whitney U test was applied to compare the differences in relative PTCH1 mRNA expression between the gastric carcinoma and adjacent normal tissues. Fisher's exact test was applied to compare the differences in PTCH1 gene promoter methylation rate between the gastric cancer and adjacent normal tissues. Spearman's test was used to study the correlation between PTCH1 methylation and its expression in gastric cancer tissues.

\section{Results}

Expression of PTCH1 mRNA in the gastric cancer AGS cell line and gastric cancer and adjacent normal tissues. The expression of the PTCH1 gene in the gastric cancer tissues and gastric cancer AGS cell line were observed by qPCR. Taking AGS as a reference sample, the relative expression levels of PTCH1 in the gastric cancer and adjacent normal tissues were $1.26 \pm 0.89$

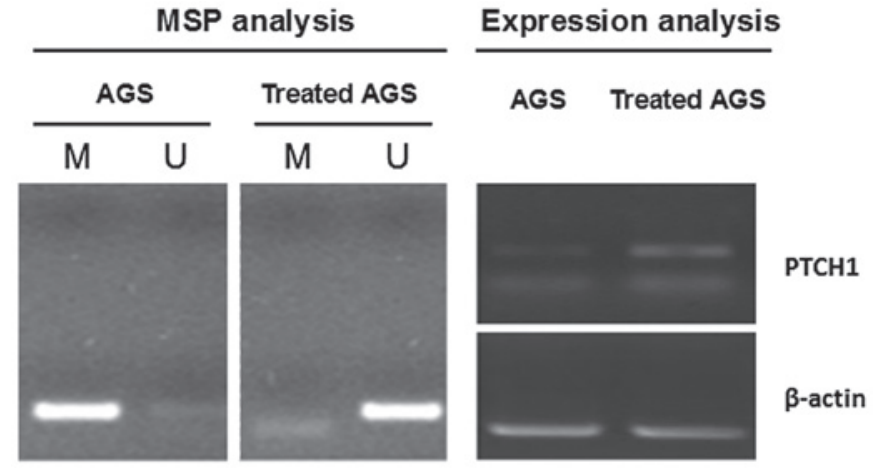

Figure 3. PTCH1 methylation and PTCH1 mRNA expression of gastric cancer AGS cells treated with 5-Aza-dC. M, methylated PCR products; U, unmethylated PCR products; MSP, methylation-specific PCR; 5-Aza-dC, 5-aza-2'-deoxycytidine.

and $2.74 \pm 1.67$, respectively. There was a significant statistical difference between the two groups $(n=20 ; P=0.023)$.

Methylation changes in the PTCH1 gene promoter region of gastric cancer and adjacent normal tissues, detected using MSP. In order to observe the incidence of PTCH1 gene methylation in the gastric cancer tissues, MSP detection was conducted in the gastric cancer and adjacent normal tissues of 20 patients. Methylation amplified bands were observed in 12 cancer tissues and 4 adjacent normal tissues, with an incident rate of $60 \%$ and $20 \%$, respectively (Fisher's exact probability test; two-tailed test $\mathrm{P}=0.057$ and one-tailed test $\mathrm{P}=0.029)$. This indicated the presence of high $\mathrm{PTCH} 1$ gene methylation in the gastric cancer tissues. Fig. 1 shows typical electrophoretograms.

Correlation between PTCH1 methylation and expression in gastric cancer tissues. In order to further study the association between PTCH1 expression and promoter methylation, the correlation between PTCH1 gene methylation and its relative 
expression in gastric cancer and adjacent normal tissues was analyzed in 20 gastric cancer patients. The qPCR values of PTCH1 in methylated and unmethylated tissues were $0.93 \pm 0.71$ and 2.58 \pm 1.52 , respectively. The expression of PTCH1 was negatively correlated with the methylation status, with a correlation coefficient of $-0.591(\mathrm{P}=0.006)$.

Impact of 5-Aza-dC treatment on the cell cycle, the apoptosis of AGS cells and the PTCH1 gene methylation status. PI staining was conducted in the AGS cells at the logarithmic phase at $72 \mathrm{~h}$ post-treatment with $5 \times 10^{-6} \mathrm{~mol} / 15-\mathrm{Aza}-\mathrm{dC}$, then the cell cycle of the treated AGS cells was analyzed (Fig. 2). A $\mathrm{G}_{0} / \mathrm{G}_{1}$ block was observed in the treated AGS cells. Meanwhile, Annexin V/PI double-staining was conducted to detect the cell apoptosis of the treated AGS cells. Significant levels of apoptosis were observed in the treated AGS cells. There were significant differences between the treated group and the control group according to the results of three independent experiments $(\mathrm{P}<0.05$; Fig. 2). MSP and relative expression measurements were conducted on PTCH1 gene methylation and expression in the AGS cells prior to and following the treatment with 5-Aza-dC AGS, as shown in Fig. 3 (4). Amplification was obtained for the methylated sequence of the PTCH1 gene, but not for the demethylated sequence in the AGS cells prior to the treatment. The relative PTCH1 mRNA expression was low in the AGS cells prior to the treatment. Amplification was obtained for the demethylated sequence of the PTCH1 gene, but not for the methylated sequence in the AGS cells following the treatment. The relative expression of the PTCH1 mRNA was increased in the AGS cells following the treatment. This further indicates the negative correlation between PTCH1 gene hypermethylation and expression.

\section{Discussion}

Cancer epigenetics studies have found that there is widespread hypomethylation and partial regional hypermethylation of $\mathrm{CpG}$ islands in the genomic DNA of cancer cells. Partial regional hypermethylation of $\mathrm{CpG}$ islands may result in the inactivation of certain tumor suppressor genes, which is an important mechanism for causing the malignant transformation of cells $(5,6)$. Demethylation agents, such as 5-Aza-dC, could be used to obtain the re-expression of these hypermethylation genes and to play a significant role in tumor suppression (7).

The $\mathrm{Hh}$ signaling pathway is a crucial signal transduction pathway in the regulation of embryonic development. The $\mathrm{Hh}$ signal is most active in the embryonic formation period, and has no expression or extremely low expression in normal mature tissues. However, aberrant activation of the Hh signal transduction pathway in cells of mature tissues and organs can result in various diseases and tumorigenesis. The high expression of SHH in small cell lung cancer tissues and cell lines has been reported in a previous study (8).

In total, $81 \%$ of tumor cell lines from the digestive tract (including the esophagus, stomach, bile duct and pancreas) have been shown to express SHH and its receptor, PTCH1 (9). The hypomethylation of HHIP, the inhibitor of $\mathrm{SHH}$, has been observed in gastrointestinal tumors (10). The missing or mutated PTCH1 gene has rarely been reported in gastric cancer in previous studies. Few studies have also reported the correlation between the methylation of the PTCH1 gene and gastric cancer. In the present study, the expression of PTCH1 mRNA was detected in gastric cancer tissues, adjacent normal tissues and a human gastric cancer cell line, with a relatively high expression of PTCH1 mRNA in the adjacent normal tissues compared with the cancer tissues. High methylation levels of PTCH1 were observed in the gastric cancer tissues and the cancer cell lines. This indicates that the high methylation modification of PTCH1 as a tumor suppressor gene may be one of the main mechanisms of gastric cancer.

In conclusion, the hypermethylation of the PTCH1 gene promoter region in gastric cancer was observed in the present study. Following this preliminary result, further studies of molecular mechanisms involved in regulating the PTCHI methylation changes and the association between PTCH1 hypermethylation and the biological features of gastric cancer are required. More scientific experimental evidence regarding PTCH1 gene hypermethylation as a gastric cancer marker and its function in guiding the treatment and prognosis of gastric cancer are also required from these studies.

\section{Acknowledgements}

This study was supported by a grant from the Open Project Program of Key Discipline for Medicine of Jiangsu Province (WKF2013-05).

\section{References}

1. Saqui-Salces M and Merchant JL: Hedgehog signaling and gastrointestinal cancer. Biochim Biophys Acta 1803: 786-795, 2010.

2. Shahi MH, Lorente A and Castresana JS: Hedgehog signaling in medulloblastoma, glioblastoma and neuroblastoma. Oncol Rep 19: 681-688, 2008.

3. Cul'bová M, Lasabová Z, Stanclová A, et al: Methylation of selected tumor-suppressor genes in benign and malignant ovarian tumors. Ceska Gynekol 76: 274-279, 2011 (In Slovak).

4. Zuo Y and Song Y: Detection and analysis of the methylation status of PTCH1 gene involved in the hedgehog signaling pathway in a human gastric cancer cell line. Exp Ther Med 6: 1365-1368, 2013.

5. Tada M, Kanai F, Tanaka Y, et al: Down-regulation of hedgehog-interacting protein through genetic and epigenetic alterations in human hepatocellular carcinoma. Clin Cancer Res 14: 3768-3776, 2008.

6. Caffarelli E and Filetici P: Epigenetic regulation in cancer development. Front Biosci (Landmark Ed) 16: 2682-2694, 2011.

7. To KF, Leung WK, Lee TL, et al: Promoter hypermethylation of tumor-related genes in gastric intestinal metaplasia of patients with and without gastric cancer. Int J Cancer 102: 623-628, 2002.

8. Watkins DN, Berman DM, Burkholder SG, et al: Hedgehog signalling within airway epithelial progenitors and in small-cell lung cancer. Nature 422: 313-317, 2003.

9. Ma X, Sheng T, Zhang Y, et al: Hedgehog signaling is activated in subsets of esophageal cancers. Int J Cancer 118: 139-148, 2006.

10. Taniguchi H, Yamamoto H, Akutsu N, et al: Transcriptional silencing of hedgehog-interacting protein by $\mathrm{CpG}$ hypermethylation and chromatic structure in human gastrointestinal cancer. J Pathol 213: 131-139, 2007. 